\title{
Expanding the utility of the dextran sulfate sodium (DSS) mouse model to induce a clinically relevant loss of intestinal barrier function
}

\author{
Kyle E. Cochran ${ }^{1}$, Nicholas G. Lamson ${ }^{1}{ }^{,}$Kathryn A. Whitehead $^{\text {Corresp. } 1,2}$ \\ 1 Department of Chemical Engineering, Carnegie Mellon University, Pittsburgh, PA, United States \\ 2 Department of Biomedical Engineering, Carnegie Mellon University, Pittsburgh, PA, United States \\ Corresponding Author: Kathryn A. Whitehead \\ Email address: kawhite@cmu.edu
}

Background. Inflammatory bowel disease (IBD) is a family of debilitating disorders that affects more than 1 million people in the United States. Many animal studies of IBD use a dextran sulfate sodium (DSS) mouse model of colitis that induces rapid and severe colitis symptoms. Although the typical seven-day DSS model is appropriate for many studies, it destroys intestinal barrier function and results in intestinal permeability that is substantially higher than what is typically observed in patients. As such, therapies that enhance or restore barrier integrity are difficult or impossible to evaluate.

Methods. We identify administration conditions that result in more physiologically relevant intestinal damage by systematically varying the duration of DSS administration. We administered 3.0\% DSS for four to seven days and assessed disease metrics including weight, fecal consistency, intestinal permeability, spleen weight, and colon length. Histology was performed to assess the structural integrity of the intestinal epithelium.

Results. Extended exposure (seven days) to DSS resulted in substantial, unrecoverable loss of intestinal structure and intestinal permeability increases of greater than 600 -fold. Attenuated DSS administration durations (four days) produced less severe symptoms by all metrics. Intestinal permeability increased only 8-fold compared to healthy mice, better recapitulating the 2-18 fold increases in permeability observed in patients. The attenuated model retains the hallmark properties of colitis against which to compare therapeutic candidates.Our results demonstrate that an attenuated DSS colitis model obtains clinically relevant increases in intestinal permeability, enabling the effective evaluation of therapeutic candidates that promote barrier function. 
1 Expanding the utility of the dextran sulfate sodium (DSS) mouse model to induce a

2 clinically relevant loss of intestinal barrier function

3 Kyle E. Cochran ${ }^{1}$, Dr. Nicholas G. Lamson ${ }^{1}$, and Dr. Kathryn A. Whitehead ${ }^{1,2 *}$

$4{ }^{1}$ Department of Chemical Engineering, Carnegie Mellon University, Pittsburgh PA 15213, USA

5 2Department of Biomedical Engineering, Carnegie Mellon University, Pittsburgh PA 15213,

6 USA

$7 \quad$ *To whom correspondence should be addressed:

8 Kathryn Whitehead

95000 Forbes Avenue, Pittsburgh, PA 15213, USA

10 Email address: kawhite@cmu.edu 


\section{Abstract}

15 Background. Inflammatory bowel disease (IBD) is a family of debilitating disorders that affects more than 1 million people in the United States. Many animal studies of IBD use a dextran sulfate sodium (DSS) mouse model of colitis that induces rapid and severe colitis symptoms. Although the typical seven-day DSS model is appropriate for many studies, it destroys intestinal barrier function and results in intestinal permeability that is substantially higher than what is typically observed in patients. As such, therapies that enhance or restore barrier integrity are difficult or impossible to evaluate.

Methods. We identify administration conditions that result in more physiologically relevant intestinal damage by systematically varying the duration of DSS administration. We administered 3.0\% DSS for four to seven days and assessed disease metrics including weight, fecal consistency, intestinal permeability, spleen weight, and colon length. Histology was performed to assess the structural integrity of the intestinal epithelium.

Results. Extended exposure (seven days) to DSS resulted in substantial, unrecoverable loss of intestinal structure and intestinal permeability increases of greater than 600 -fold. Attenuated DSS administration durations (four days) produced less severe symptoms by all metrics. Intestinal permeability increased only 8 -fold compared to healthy mice, better recapitulating the 2-18 fold increases in permeability observed in patients. The attenuated model retains the hallmark properties of colitis against which to compare therapeutic candidates. Our results demonstrate that an attenuated DSS colitis model obtains clinically relevant increases in intestinal permeability, enabling the effective evaluation of therapeutic candidates that promote barrier function. 


\section{Introduction}

Inflammatory bowel disease (IBD) is a set of painful and debilitating disorders that affects approximately 1.6 million Americans, with upward of 70,000 new diagnoses each year. ${ }^{1-3}$ IBD includes ulcerative colitis, Crohn's disease, and indeterminant colitis, and our understanding of the etiology of these diseases is lacking. Patients with IBD suffer from persistent diarrhea, rectal bleeding, weight loss, fatigue, and abdominal pain, which dramatically impacts their health and quality of life. ${ }^{4}$ Current treatments manage, rather than cure, IBD and include antibiotics, steroids, and immunosuppressants. ${ }^{5,6}$ Although the full mechanisms through which the disease develops remain uncertain, evidence points to loss of intestinal barrier function as a key aspect of clinical IBD. It is unclear if barrier loss is a symptom or a cause of the disease. ${ }^{7,8}$ Thus, reducing intestinal permeability has been the goal of some therapeutic candidates, ${ }^{9}$ and the success of such studies is dependent on the use of appropriate animal models.

The most widely used IBD model uses dextran sulfate sodium (DSS) that is introduced into the drinking water of mice to chemically induce colitis. Although its exact mechanism for inducing colitis is unknown, DSS administered in the drinking water of mice inflicts damage to the intestinal epithelium. ${ }^{10,11}$ This results in colitis symptoms including loose and bloody stool, significant weight loss, shortening of the colon, and decreased intestinal barrier function. ${ }^{10,12,13}$ This model is the current standard in many laboratories because it is relatively reproducible, customizable, and doesn't require the spontaneous development of colitis that models using transgenic or knockout mice do. ${ }^{12}$ However, a significant downfall of the model is the rapid and widespread damage to the intestinal epithelium, which doesn't reflect the degree of decreased barrier function in patients. ${ }^{11}$ 
60

to eight days. ${ }^{14,15}$ While this falls within the recommendations of administration protocols, ${ }^{10}$ the rapid and severe colitis and epithelial damage induced by these models does not resemble clinical IBD. ${ }^{11}$ Specifically, these models result in loss of intestinal barrier function that is far more severe than the losses observed in clinic. Lactulose/mannitol tests have demonstrated intestinal permeability increases of 2- to 18 -fold in colitis patients, while the standard DSS model can increase permeability to macromolecule markers by more than 100 -fold. ${ }^{16,17}$ Studies of therapeutics that restore intestinal barrier function have been limited, likely in part because the standard DSS model destroys the intestinal epithelial monolayer. Fortunately, previous research suggests that it is possible to induce milder colitis symptoms by reducing DSS administration duration. ${ }^{18}$ We were motivated to expand upon these findings by evaluating a broad array of colitis metrics, including intestinal permeability, in an attenuated DSS mouse model.

To identify DSS colitis protocol conditions that better recapitulate the loss of barrier function present in clinical IBD, we fixed the DSS dose at 3.0\% and varied exposure length from four to seven days. We tracked the onset and progression of weight loss, fecal samples, colon length, spleen weight, and intestinal permeability and scored colon histological samples. In addition to identifying DSS administration conditions that result in clinically relevant levels of intestinal permeability, we also examined how environmental factors, such as acclimation to experimental conditions, affected colitis onset. 


\section{Materials and methods}

\section{Animals}

84
Animal protocols were approved by the Institutional Animal Care and Use Committee at Carnegie Mellon University (Pittsburgh, PA), and all experiments were conducted in accordance with approved protocol AR201900009. Furthermore, all studies were conducted in accordance with local, institute, and federal regulations for vertebrate animal research. Female C57BL/6 mice six weeks of age were purchased from Charles River Laboratories and acclimated to facility conditions for two weeks before studies. Mice were housed under controlled temperature $\left(25^{\circ} \mathrm{C}\right)$ on 12 hour light-dark cycles. Animals were given access to standard diet and water, with experimental groups receiving 3\% DSS in their water. Animals were euthanized early if they lost more than $30 \%$ of their initial body weight or if they showed severe signs of distress. All animals were euthanized at the end of the study by $\mathrm{CO}_{2}$ inhalation.

\section{Materials}

Dextran sodium sulfate colitis grade (DSS, 36,000-50,000 MW) was purchased from MP Biomedicals (Santa Ana, CA). Fluorescein isothiocyanate-dextran 4 kDa (FITC-DX4) was purchased from Sigma Aldrich (St. Louis, MO). Hemoccult Guaiac Fecal Occult Blood Test slides were purchased from VWR (Radnor, PA). Phosphate buffered saline (PBS) was purchased from Thermo Fisher LifeTech (Carlsbad, CA). Water bottles, spouts, cages, and bedding were provided by Mellon Institute Centralized Vivarium.

\section{DSS Administration}


104 Glass water bottles with stainless steel drinking spouts were filled with $100 \mathrm{~mL}$ of chlorine 105 treated water and placed in each group's cage. For groups receiving DSS, 3.0 grams of DSS was 106 fully dissolved in the water before the bottle was placed the cage. Water level for each cage was 107 recorded daily and the water was fully changed every two days. Animals received DSS for 7, 6, 1085 , or 4 days, after which they were switched to fresh water for 7 days unless early euthanasia was 109 required. Groups were designed so that DSS administration was staggered and all animals were 110 switched to fresh water on the same day.

112 Weight and Fecal Scores

113 Weight was recorded and a small fecal sample was collected for each animal daily. Fecal 114 samples were examined for consistency, tested for blood using Hemoccult slides, and scored 115 accordingly.

\section{Intestinal Permeability}

118 Mice were rectally administered FITC-DX4 as a colonic permeation marker $(60 \mathrm{mg} / \mathrm{mL}$ in PBS, $119600 \mathrm{mg} / \mathrm{kg}$ animal). Three hours later, blood samples were taken via the submandibular gland and the serum was examined for fluorescence at 495/519 nm on a BioTek Synergy2 plate reader. Application of a calibration curve yields a blood concentration of FITC-DX4.

\section{Evaluation of Colons and Spleens}

124 Upon euthanasia, the spleen and colon of each animal were excised. Colons were measured to 125 the nearest half millimeter then fixed in $4 \%$ formaldehyde for 24 hours. Fixed colons were 126 washed twice with PBS and subsequently stored in $70 \%$ ethanol at $25^{\circ} \mathrm{C}$. Hematoxylin and eosin 
127 (H\&E) staining was performed on colon cross-sections by University of Pittsburgh Medical

128 Center Tissue and Research Pathology Services (Pittsburgh, PA). Stained slides were scored and 129 imaged by Dr. Lora Rigatti, University of Pittsburgh (Pittsburgh, PA). Spleens were weighed on 130 an Ohause Adventure Pro analytical balance.

132 Statistical Analysis

133 Animals were placed in group of 6 per cage, and premature animal deaths were accounted for in 134 analysis. Error is displayed in all plots as the standard error of the mean and experimental 135 groups are compared to the control individually using ordinary t-test, without correction for 136 multiple comparisons.

\section{Results}

The use of an animal model that recapitulates key aspects of colitis is crucial to the study of disease mechanisms and evaluation of potential IBD therapeutics. One of the most common colitis models, the DSS model, is highly tunable and can be used to induce colitis of varying severities. We reasoned, therefore, that it would be possible to induce colitis symptoms without destroying intestinal barrier function.

\section{Establishing a Model with Less Severe Symptoms}

147 To identify a chemically-induced colitis protocol that results in less severe, yet measurable, 148 symptoms, we examined how altering the administration duration of DSS affected symptom 149 onset. To accomplish this, groups received 3.0\% DSS in their drinking water for either seven, 
150 six, five, or four days. After the DSS administration period, they were switched to fresh water

151 for seven days, or four days if early euthanasia was required. Starting with the seven-day group

152 on Day 0, each group began receiving DSS on subsequent days, such that all animals were

153 switched to fresh water on Day 7 (Fig 1a). Each day, mice were weighed, and fecal samples

154 were collected and scored. All DSS animals began losing substantial weight four to five days

155 after the start of DSS administration (Fig 1b), but only the 4-day DSS group made any recovery

156 before euthanasia was required. The seven, six, and five-day groups were unable recover body

157 weight, and each required early euthanasia because they reached $30 \%$ weight loss (a

158 predetermined endpoint of the study). Average weight loss per day was calculated between the

159 fourth and eighth day of DSS administration. Average daily weight loss was similar between

160 colitis groups, but animals from the four-day DSS group lost less weight per day than animals

161 from the five-day DSS group $(\mathrm{p}=0.0068$, Fig 1c).

162

163 Each day, fecal samples were taken and scored for consistency and blood content (Fig 1d and Fig

164 1e). Colitis is indicated by the increasing fecal scores observed for all DSS groups. Similar to

165 weight loss, the onset of increasing fecal scores was similar across all colitis groups, but only the

166 4-day DSS group recovered after returning to fresh water. Together, these results indicate that

167 shortening the DSS administration time affected the severity, rather than the onset, of colitis

168 symptoms such as weight and fecal score.

\section{Effect of DSS Administration Duration on Intestinal Permeability}

171 Decreased intestinal barrier function is a symptom of clinical IBD and IBD animal models. ${ }^{10,19}$

172 To demonstrate the loss of intestinal barrier function during colitis development, we rectally 
173 administered the permeability marker FITC-DX4 (4 kDa) on Days 7, 10, and 14 and collected

174 blood samples three hours later. The concentration of this fluorescent permeability marker in

175 serum samples was analyzed to assess the barrier function of the animals' colons.

176

177 On Day 7, the day on which each group completed their DSS regimen and was switched to fresh

178 water, the seven $(p=0.005)$, six $(p<0.0001)$, and five-day $(p<0.0001)$ DSS groups each had

179 significantly elevated intestinal permeabilities (Fig 2). The four-day DSS group had

180 permeabilities that were slightly higher than the control, while the seven-day DSS group showed

181 the most intense increase in permeability, increasing 35-fold compared to the control.

182 Permeability measurements on Day 10, three days after animals were switched to fresh water,

183 showed that barrier function continues to decline even when DSS is discontinued. At this point,

184 the seven-day group showed a greater than 600-fold increase in intestinal permeability compared

185 to the control $(p=0.0063)$, while the six $(p=0.1364)$, five $(p=0.0936)$, and four-day $(p=$

186 0.0089) DSS groups all had less than a 22-fold increase (Fig 2).

187

188 Because animals from the seven, six, and five-day DSS groups reached greater than $30 \%$ body

weight loss around Day 10, they required early euthanasia. Only the control and 4-day DSS

groups continued through the entire 14-day experiment. On Day 14, the final intestinal

permeability measurement for the four-day DSS group showed that animals had returned to near

192

control values, indicating a restoration of colonic barrier function (Fig 2). Overall, the

193

abbreviated 4-day DSS protocol results in a measurable increase in intestinal permeability

without permanently destroying intestinal barrier function. 
196

197

198

199

200

201

202

203

204

205

206

207

208

209

210

211

212

213

214

215

216

217

218

\section{Effect of DSS Administration Duration on Spleen Weight and Colon Length}

As colitis progresses and the epithelium begins to erode, the colon thins and becomes shorter and immune cells infiltrate the lamina propria. ${ }^{10}$ Infiltration of immune cells results in inflammation that is often assessed by measuring spleen weight. These phenomena provide two additional metrics, colon length and spleen weight, by which the severity of chemically-induced colitis can be measured. ${ }^{10}$ To examine these aspects of the disease model, mice were euthanized on the final day of the experiment and their colons and spleens were harvested. Spleen weights from all groups differed significantly from the control $(p=0.0003$ for seven-day, $p=0.0008$ for six-day, $p=0.0127$ for five-day, $p=0.0047$ for four-day), but only the four-day DSS group experienced enlarged spleens (Fig 3a). The seven, six, and five-day DSS groups each had spleen weights that were significantly less than the control. Dehydration and lack of a recovery (requiring early euthanasia) are likely the reasons that groups receiving DSS for longer durations had lower spleen weights.

The colons of animals from all DSS groups were significantly shorter than control animals $(\mathrm{p}<$ 0.0001 for all groups, Fig 3b). Although the symptoms of the four-day DSS protocol were less severe, thinning and shortening of the colon nonetheless occurs. This indicates that colonic shortening, one of the hallmarks of chemically induced colitis, is retained in this attenuated fourday model and provides a viable metric against which to compare IBD therapeutics.

\section{Histological Evaluation of Colon Samples from the Colitis Model}

To assess the effect that DSS administration duration has on the degree of damage to the intestinal epithelium, three colon samples from each group were stained with hematoxylin and 
219 eosin (H\&E) and scored. Each sample was blindly scored by a trained pathologist for four

220 criteria shown in Fig. 4a: inflammation (0-3), extent (0-3), regeneration (3-0), and crypt damage

221 (0-4). The score from each criterion was multiplied by a score for the involvement (0-3) to

222 indicate how much of the sample section was affected. Possible scores ranged from 0-39, with

223 higher scores indicating increased damage to the intestinal epithelium.

225 Control mice had an average score of 0 , indicative of the fully intact epithelium found in healthy mice (Fig 4b). Mice that received DSS had individual scores ranging from 8 to 36, and scores

227

228

229

230

231

232

233

234

235

236

237

238

239

240

241 generally increased with increasing length of DSS administration. The 7-day DSS group had the highest score with an average of $32.5(\mathrm{p}<0.0001)$, followed by the 6-day DSS group with an average of $19.7(\mathrm{p}<0.0001)$. The five- and four-day DSS groups had the lowest average histological scores, $17.3(\mathrm{p}=0.0319)$ and $18.7(\mathrm{p}=0.0996)$, respectively. Images of selected colons in Fig. 4c show the increasing loss of epithelial structure as DSS administration duration increased. In the seven- and six-day DSS groups, lack of crypts further confirms the severity of the colitis associated with longer DSS administration regimens. Similar to other metrics, the histological scores show that the full seven-day DSS model resulted in substantial damage, unrecoverable to the intestinal epithelium. Reducing DSS administration time decreased the severity of the inflicted damage, although there was some variability within groups.

\section{Avoiding Pitfalls: Acclimation to Experimental Conditions}

Chemically-induced colitis affects each animal differently, which manifests as highly variable results in these models. To reduce variability, animals should be given ample time to acclimate to experimental conditions before observations begin. In our animal facility, DSS administration 
242 required removal of the standard Lixit drinking system, which was replaced with individual

243 water bottles in each cage. Animals must be properly acclimated to this switch before

244 experimental observations begin.

245

246 To demonstrate this, we set up control and DSS cages that were switched from the Lixit system

247 to water bottles five days before observations (Day -5) or on the day that observation began (Day

248 0) (Fig 5a and b). For control animals that were switched to water bottles on Day 0 (Fig 5a),

249 there was a significant drop off in weight of about $3 \%$ on Day $1(p=0.0080)$, likely due to

250 hesitation by the mice to drink water from an unfamiliar system. In general, the weight of these

251 mice was less consistent than that of the mice that were acclimated to water bottles five days

252 before observations began. A similar pattern was observed for mice receiving DSS. On Day 1,

253 mice that were not acclimated to the water bottle lost an average of $5 \%$ of their body weight

254 (blue squares, Fig 5b). Although these mice did recover that weight, their initial lack of water

255 consumption resulted in a measurable delay in the onset of colitis related weight loss compared

256 to acclimated mice (green circles). On each day after Day 4, acclimated mice had significantly

257 lower weights compared to the control, $(p=0.0060$ for Day $4, p=0.0065$ for Day $5, p=0.0288$

258 for Day 6, and $\mathrm{p}=0.0129$ for Day 7) indicating the faster and more consistent colitis onset. For

259 this reason, we recommend that mice be acclimated to experimental conditions, including

260 drinking from water bottles, at least five days prior to the experiment to allow them ample time

261 to adjust.

262

263 Discussion 
As IBD becomes increasingly prevalent and ever-growing efforts are devoted to

understanding and treating the disease, it is imperative to fully understand IBD models and how to best implement them. DSS is often administered at high doses for longer durations to induce an acute colitis. ${ }^{14,15}$ Our results confirm that this induces a sweeping loss of intestinal barrier function, which precludes the testing of therapeutics designed to repair the intestinal epithelium. Decreased intestinal barrier function is a critical factor in clinical IBD and the DSS colitis model due to the subsequent immune cell infiltration and inflammatory response. ${ }^{12,19}$ However, DSS administration inflicts widespread damage to the intestinal epithelium that results in permeability increases far higher than those observed in clinical IBD. ${ }^{16,17}$ Permeability increased in all groups receiving DSS, but the magnitude of the increase was predicated on administration duration. The four-day DSS protocol produced intestinal permeability changes that best mimic what is observed clinically. First, the 8 -fold increase in intestinal permeability for the four-day group best recapitulates the 2 - to 18 -fold increases observed in IBD patients ${ }^{16,17}$. Furthermore, permeability in this group returned to baseline before euthanize. These results are more representative of many cases of clinical IBD where a patient experiences bouts of inflammation from which they can recover. Although the 5-day group experienced similar increases in permeability (10-fold compared to control animals), their body weight loss required early euthanize. In experiments in which epithelial treatments are evaluated, this early endpoint would limit the assessment of the therapeutic since it cannot be administered through symptom resolution. It will be necessary for other research laboratories to run their own attenuated protocols to assess whether 5, 4, or potentially even 3-day DSS administration regimens are most appropriate for their specific mouse colonies and their specific batch of DSS, which can vary in potency. 
Although not a direct measure of inflammation, recording spleen weight at euthanasia is a

288

289

290

291

292

293

294

295

296

297

298

299

300

301

302

303

304

305

306

307

308

309

quick way to correlate inflammation to DSS administration. ${ }^{10}$ As colitis progresses in the

animal, the resulting immune response leads to an increase of immune cells and thus an increase

in spleen weight. The increased weight of the spleens from the four-day DSS group is indicative

of the robust immune response present despite abbreviating the colitis induction period.

Conversely, the decrease in spleen weight observed in the seven, six, and five-day DSS groups is

likely the result of the severe dehydration and weight loss experienced by these animals.

Shortening of the colon is a hallmark of chemically induced colitis and an often-used

metric to compare IBD treatments to controls. ${ }^{10}$ All groups from this work demonstrated

significant colonic shortening, regardless of administration duration. These data indicate that,

despite inducing less severe colitis by most metrics, the four-day DSS model retains measurable colitis symptoms against which to compare therapeutics.

Histological scoring also confirmed that the 4-day DSS model produced measurable symptoms of colitis. All DSS groups showed epithelial damage and inflammation compared to the control group, with severity corresponding to administration duration. Histological scores indicate that shorter DSS administration duration generally results in less severe damage to the intestinal epithelium.

Although the etiology of clinical IBD remains unclear, it is generally accepted that genetic predisposition is not the only contributing factor. Diet and lifestyle of western societies are inferred to have some responsibility for the increased prevalence of IBD in countries such as the United States. ${ }^{1-3}$ The DSS colitis model is similar in that environmental factors, including diet and animal facility conditions, impact the induced colitis. ${ }^{12}$ This makes comparing results between different studies difficult and leads to increased variability of the model, but measures 
310 can be taken to ensure that experiments are reproducible with steady colitis induction. All

311 animals in the experiment should be properly acclimated to the animal housing facility before

312 colitis induction begins. Because DSS must be administered via drinking water, the acclimation

313 period should include training animals to drink from the water bottle, rather than the system-

314 wide fresh water supply. Failing to properly acclimate animals results in altered disease model

315 symptoms, which confounds weight loss due to colitis, stress, and/or dehydration.

316 This study is limited to measurements of weight, fecal scores, intestinal permeability,

317 colon length, spleen weight, and histological evaluation. IBD is a complex disease, and it is

318 likely important to consider other factors, such as tight junction protein expression, the

319 microbiota, and cytokine production. ${ }^{7}$ The data presented here suggest that this attenuated four-

320 day DSS model produces results that better recapitulate clinical increases in intestinal

321 permeability. A more robust analysis should be done to confirm that other markers in this model

322 sufficiently represent the clinical disease state.

323

\section{Conclusion}

325 The DSS colitis model is a powerful tool for studying disease mechanisms and potential

therapeutics for IBD. Administering DSS for seven days results in the rapid onset of severe colitis that precludes the testing of epithelium-focused treatments. Here, we demonstrate that an attenuated four-day model induces increases in intestinal permeability that better mimic those of IBD patients while retaining the hallmark signs of chemically-induced colitis. Because this model does not destroy the intestinal epithelium, it will enable the screening of therapeutics that aim to restore or improve barrier function in colitis patients. 
334 The authors also thank M. Oudhoff and J. Gleeson for their helpful discussions and input. Any

335 opinions, findings, and conclusions or recommendations expressed in this material are those of the

336 author(s) and do not necessarily reflect the views of the National Science Foundation.

337

338

339

340

341

342

2. Kaplan GG. The global burden of IBD: from 2015 to 2025. Nat Rev Gastroenterol

\section{REFERENCES}

1. Loftus E V. Clinical epidemiology of inflammatory bowel disease: Incidence, prevalence, and environmental influences. Gastroenterology. 2004;126(6):1504-1517. doi:10.1053/j.gastro.2004.01.063

3. Colombel J-F, Mahadevan U. Inflammatory Bowel Disease 2017: Innovations and Changing Paradigms. Gastroenterology. 2017;2(152):309-312.

4. Strober W, Fuss I, Mannon P. The fundamental basis of inflammatory bowel disease. Sci Med. 2007;117(3):514-521. doi:10.1172/JCI30587.514

5. Pithadia AB, Jain S. Treatment of inflammatory bowel disease (IBD ). Pharmacol Reports. 2011;63(3):629-642. doi:10.1016/S1734-1140(11)70575-8

6. Triantafillidis JK, Merikas E, Georgopoulos F. Current and emerging drugs for the treatment of inflammatory bowel disease. Drug Des Devel Ther. 2011;5:185-210. doi:10.2147/DDDT.S11290

7. Martini E, Krug SM, Siegmund B, Neurath MF, Becker C. Mend Your Fences: The Epithelial Barrier and its Relationship With Mucosal Immunity in Inflammatory Bowel Disease. Cmgh. 2017;4(1):33-46. doi:10.1016/j.jcmgh.2017.03.007

8. Lee J, Wasinger V, Yau Y, Chuang E, Yajnik V, Leong R. Molecular Pathophysiology of Epithelial Barrier Dysfunction in Inflammatory Bowel Diseases. Proteomes. 2018;6(2):17.

9. Arrieta MC, Madsen K, Doyle J, Meddings J. Reducing small intestinal permeability attenuates colitis in the IL10 gene-deficient mouse. Gut. 2009;58(1):41-48. doi:10.1136/gut.2008.150888

10. Chassaing B, Aitken JD, Malleshappa M, Vijay-Kumar M. Dextran sulfate sodium (DSS)induced colitis in mice. Curr Protoc Immunol. 2014;(SUPPL.104):1-14. doi:10.1002/0471142735.im1525s 104 
Hyg (Geneve). 2001;59(2332):241-248. doi:10.1016/j.jcmgh.2015.01.006

12. Eichele DD, Kharbanda KK. Dextran sodium sulfate colitis murine model: An indispensable tool for advancing our understanding of inflammatory bowel diseases pathogenesis. World J Gastroenterol. 2017;23(33):6016-6029. doi:10.3748/wjg.v23.i33.6016

13. Mallon P, Mansor S, Solomon L, Loughrey M, Gardiner K, Kirk S, Donnelly, E, Hoper M. The dextran sulphate sodium (DSS) model of colitis: an overview. Comp Clin Path. 2010;19(3):235-239. doi:10.1007/s00580-010-0979-4

14. Zhang S, Langer R, Traverso G, Cao B, Vermula P, Glickman J, Karp J, Hamilton M, Ermann J, Succi M, Korzenik J, Glimcher L, Zhou A. An inflammation-targeting hydrogel for local drug delivery in inflammatory bowel disease. Sci Transl Med. 2015;7(300):300ra128-300ra128. doi:10.1126/scitranslmed.aaa5657

15. He X, Wei Z, Wang J, Kou J, Liu W, Fu Y, Yang Z. Alpinetin attenuates inflammatory responses by suppressing TLR4 and NLRP3 signaling pathways in DSS-induced acute colitis. Sci Rep. 2016;6(June):1-11. doi:10.1038/srep28370

16. Welcker K, Martin A, Kölle O, Siebeck M, Gross M. Increased intestinal permeability in patients with inflammatory bowel disease. Eur J Med Res. 2004;9(10):456-460.

17. May GR, Sutherland LR, Meddings JB. Is small intestinal permeability really increased in relatives of patients with Crohn's disease? Gastroenterology. 1993;104(6):1627-1632. doi:10.1016/0016-5085(93)90638-S

18. Yan Y, Kolachala V, Dalmasso G, Nguyen H, Laroui H, Shanthi V. Temporal and Spatial Analysis of Clinical and Molecular Parameters in Dextran Sodium Sulfate Induced Colitis. PLoS One. 2009;4(6):e6073. doi:10.1371/journal.pone.0006073

19. Michielan A, Incà RD. Intestinal Permeability in Inflammatory Bowel Disease : Pathogenesis , Clinical Evaluation, and Therapy of Leaky Gut. Mediators Inflamm. 2015;2015:1-10. doi:10.1155/2015/628157

\section{Figure captions}

Fig 1. Weight loss and fecal scores were affected by the duration of DSS exposure. (A) DSS

was administered to the 7-day DSS group on D0, followed by the 7-day group on D1, and so on.

Early euthanasia was required for mice in the 7-, 6-, and 5-day DSS groups. (B) Mice from each group began losing weight four to five days after the start of their DSS regimen. Weight loss from all groups continued after switching to fresh water, with all but the 4-day DSS group 
reaching the $30 \%$ weight loss cutoff. Only the 4-day DSS group recovered body weight. (C)

400

401

402

403

404

405

406

407

408

409

410

411

412

413

414

415

416

417

418

419

420

421

Average daily weight loss was calculated for each animal, between the fourth and eighth days of their DSS regimen. Weight loss was similar across groups, with the four-day group losing the least weight per day. (D) Fecal scores increased as the DSS administration duration lengthened. (E) Fecal samples were scored daily and ranged from 0-4, with 4 indicating the most severe symptoms. Error bars for all panels display s.e.m. $(n=5-6)$.

Fig 2. DSS colitis increased intestinal permeability, the severity of which depended on DSS exposure duration. Intestinal permeability measured at Day 7 was significantly increased for all groups except the four-day DSS group. By Day 10, the permeability of the seven-day DSS group was nearly 28 -fold higher than any other group. Seven days after being switched to fresh water (Day 14), the intestinal permeability of the four-day DSS group returned to normal. Mice that received DSS for only four days experienced a significant increase in permeability but recovered to baseline after seven days of fresh water. $* \mathrm{p}<0.05, * * \mathrm{p}<0.01 * * * * \mathrm{p}<0.0001$ compared to the PBS control, error bars display s.e.m. $(n=5-6)$.

\section{Fig 3. Only animals from the 4-day DSS group experienced an increase in spleen weight,} but all DSS groups had significant shortening of the colon. (A) The spleens from all other groups weighed significantly less than the control group, likely due to the animals' severe dehydration and a lack of recovery from colitis. (B) Although a four-day DSS regimen resulted in less severe symptoms by most metrics, it nonetheless produced measurable colitis. For both panels, $* \mathrm{p}<0.05, * * * * \mathrm{p}<0.0001$, error bars display s.e.m. $(\mathrm{n}=5-6)$. 
422 Fig 4. DSS administration duration dramatically affected the integrity of the colonic

423 epithelium. (A) Colon samples were evaluated for inflammation, extent, regeneration, crypt

424 damage, and involvement and scored 0-39. Increasing scores indicate increasing damage

425 inflicted from DSS administration. (B) Epithelial and crypt structure were absent from the colon

426 samples of animals exposed to 7 days of DSS. Colonic structure gained integrity as the duration

427 of DSS exposure was reduced. (C) Colon samples were H\&E stained and representative images

428 were captured at 10x and 20x magnification (scale bars represent $100 \mu \mathrm{m}$ ).

Fig 5. Failing to acclimate animals to experimental conditions resulted in significant

differences in symptom onset. (A) When not acclimated to water bottles, control mice

(receiving only water) lost up to $3 \%$ of their body weight when they were switched from Lixit to bottle water consumption. (B) DSS-treated mice not acclimated to water bottles experienced greater fluctuations in body weight and delayed onset of symptoms compared to acclimated mice. For all panels, $\mathrm{n}=5-6$, with error bars representing s.e.m., $*=\mathrm{p}<0.05$. 


\section{Figure 1}

Weight loss and fecal scores were affected by the duration of DSS exposure.

(A) DSS was administered to the 7-day DSS group on D0, followed by the 7-day group on D1, and so on. Early euthanasia was required for mice in the 7-, 6-, and 5-day DSS groups. (B) Mice from each group began losing weight four to five days after the start of their DSS regimen. Weight loss from all groups continued after switching to fresh water, with all but the 4-day DSS group reaching the $30 \%$ weight loss cutoff. Only the 4-day DSS group recovered body weight. (C) Average daily weight loss was calculated for each animal, between the fourth and eighth days of their DSS regimen. Weight loss was similar across groups, with the four-day group losing the least weight per day. (D) Fecal scores increased as the DSS administration duration lengthened. (E) Fecal samples were scored daily and ranged from $0-4$, with 4 indicating the most severe symptoms. Error bars for all panels display s.e.m. $(n=$ $5-6)$. 
A)

control

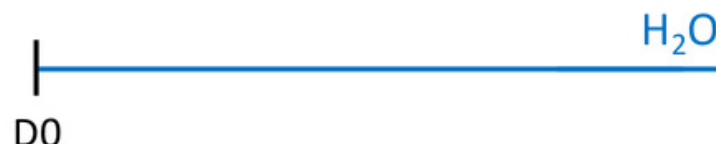

evaluation

D0

D14

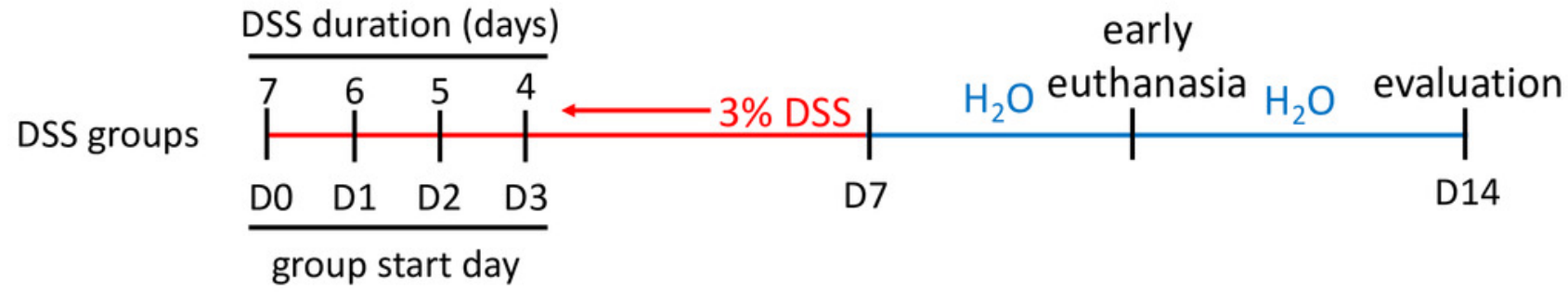

B)

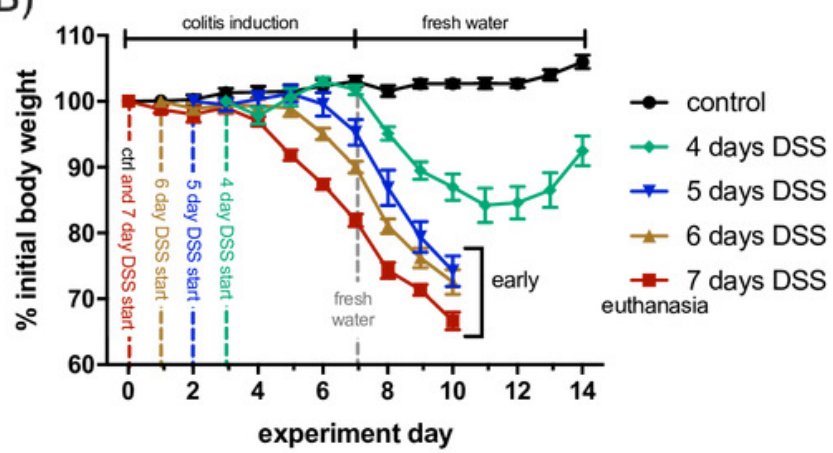

D)

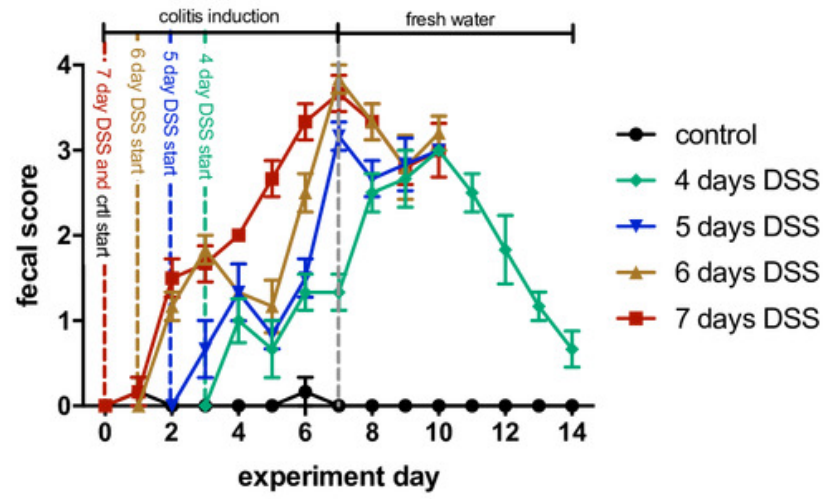

C)

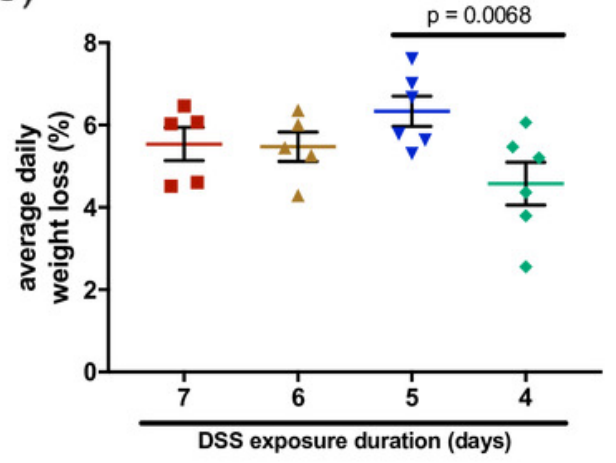

E)

fecal score criteria

$0=$ solid stool, negative hemoccult

$1=$ soft or runny stool, negative hemoccult OR solid stool with positive hemoccult

2 = soft stool, positive hemoccult

$3=$ runny or mucusy stool, positive hemoccult

$4=$ visible rectal bleeding 
Figure 2

DSS colitis increased intestinal permeability, the severity of which depended on DSS exposure duration.

Intestinal permeability measured at Day 7 was significantly increased for all groups except the four-day DSS group. By Day 10, the permeability of the seven-day DSS group was nearly 28-fold higher than any other group. Seven days after being switched to fresh water (Day 14), the intestinal permeability of the four-day DSS group returned to normal. Mice that received DSS for only four days experienced a significant increase in permeability but recovered to baseline after seven days of fresh water. $* p<0.05, * * p<0.01 * * * * p<$ 0.0001 compared to the PBS control, error bars display s.e.m. $(n=5-6)$.

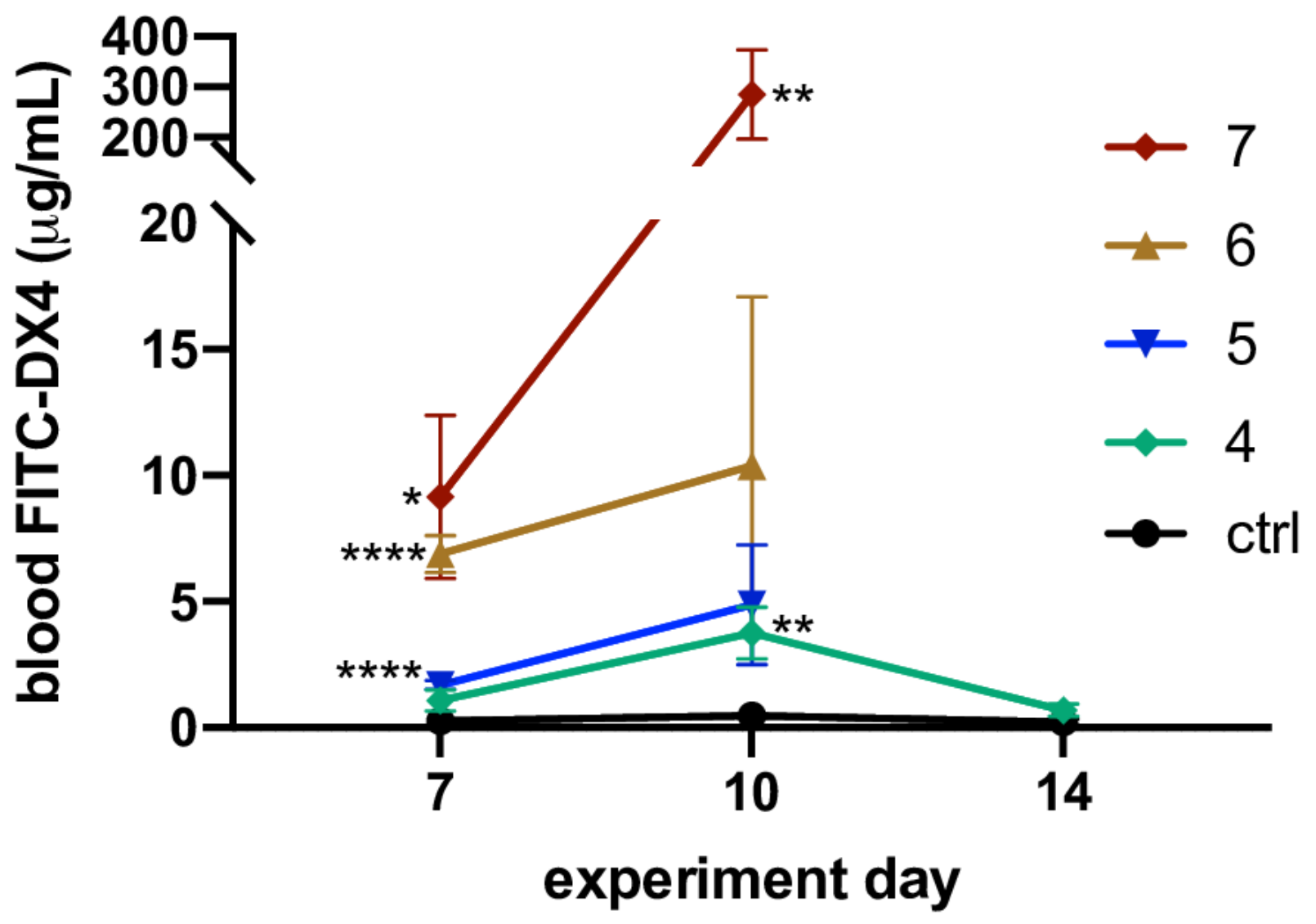


Figure 3

Only animals from the 4-day DSS group experienced an increase in spleen weight, but all DSS groups had significant shortening of the colon.

(A) The spleens from all other groups weighed significantly less than the control group, likely due to the animals' severe dehydration and a lack of recovery from colitis. (B) Although a four-day DSS regimen resulted in less severe symptoms by most metrics, it nonetheless produced measurable colitis. For both panels, $* p<0.05, * * * * p<0.0001$, error bars display s.e.m. $(n=5-6)$.

A)

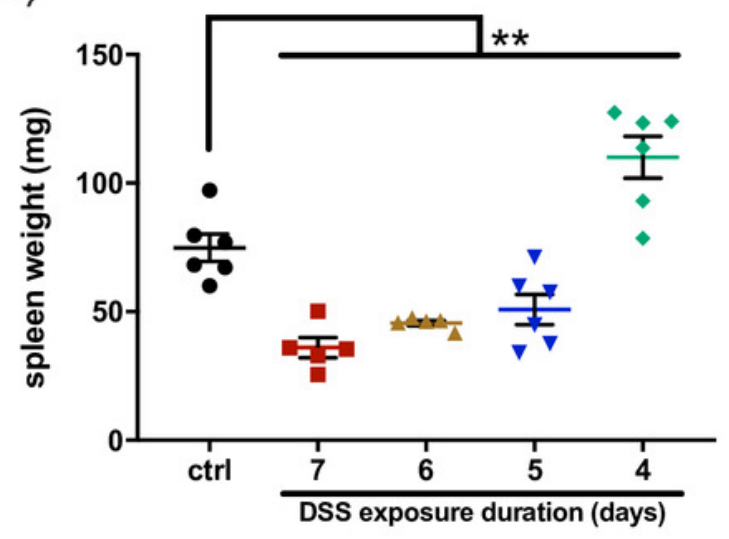

B)

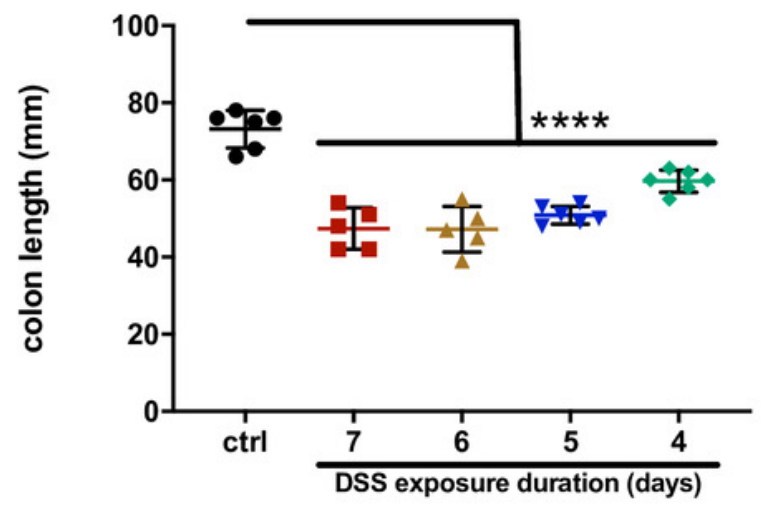


Figure 4

DSS administration duration dramatically affected the integrity of the colonic epithelium.

(A) Colon samples were evaluated for inflammation, extent, regeneration, crypt damage, and involvement and scored 0-39. Increasing scores indicate increasing damage inflicted from DSS administration. (B) Epithelial and crypt structure were absent from the colon samples of animals exposed to 7 days of DSS. Colonic structure gained integrity as the duration of DSS exposure was reduced. (C) Colon samples were H\&E stained and representative images were captured at $10 x$ and $20 x$ magnification (scale bars represent 100 $\mu \mathrm{m})$.

A)

\begin{tabular}{|c|c|c|}
\hline \multicolumn{3}{|c|}{ Scoring System } \\
\hline \multirow{4}{*}{ Inflammation } & 0 & None \\
\hline & 1 & Slight \\
\hline & 2 & Moderate \\
\hline & 3 & Severe \\
\hline \multirow{4}{*}{ Extent } & 0 & None \\
\hline & 1 & Mucosa \\
\hline & 2 & Mucosa and submucosa \\
\hline & 3 & Transmural \\
\hline \multirow{4}{*}{ Regeneration } & 3 & No tissue repair \\
\hline & 2 & Minimal regenerative change \\
\hline & 1 & Partial regeneration of gland \\
\hline & 0 & Complete regeneration or normal tissue \\
\hline \multirow{5}{*}{ Crypt Damage } & 0 & None \\
\hline & 1 & Basal $1 / 3$ damaged \\
\hline & 2 & Basal 2/3 damaged \\
\hline & 3 & Only surface epithelium intact \\
\hline & 4 & Entirecrypt and epithelium lost \\
\hline \multirow{4}{*}{ Involvement } & 0 & None \\
\hline & 1 & up to $1 / 3$ of the section \\
\hline & 2 & $1 / 3$ to $2 / 3$ of the section \\
\hline & 3 & $2 / 3$ of the section to diffuse \\
\hline
\end{tabular}

C)
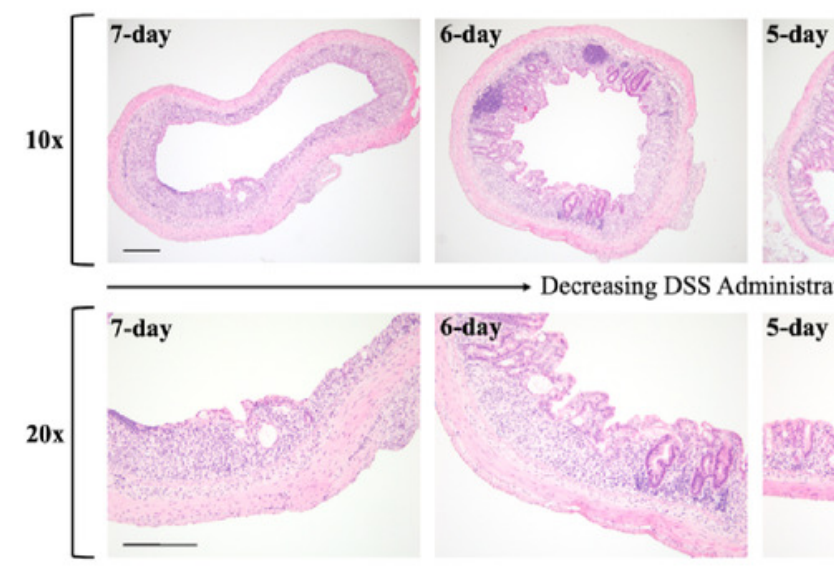

B)
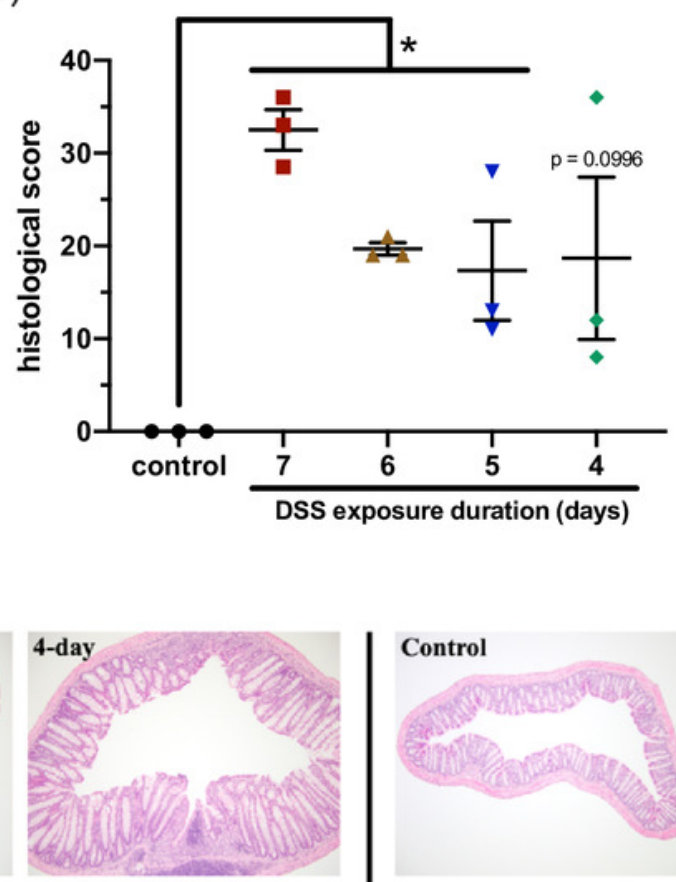

4-day

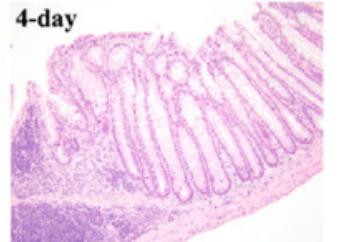

Control

Control

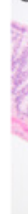


Figure 5

Fig 5. Failing to acclimate animals to experimental conditions resulted in significant differences in symptom onset.

(A) When not acclimated to water bottles, control mice (receiving only water) lost up to $3 \%$ of their body weight when they were switched from Lixit to bottle water consumption. (B) DSStreated mice not acclimated to water bottles experienced greater fluctuations in body weight and delayed onset of symptoms compared to acclimated mice. For all panels, $n=5-6$, with error bars representing s.e.m., $*=p<0.05$.
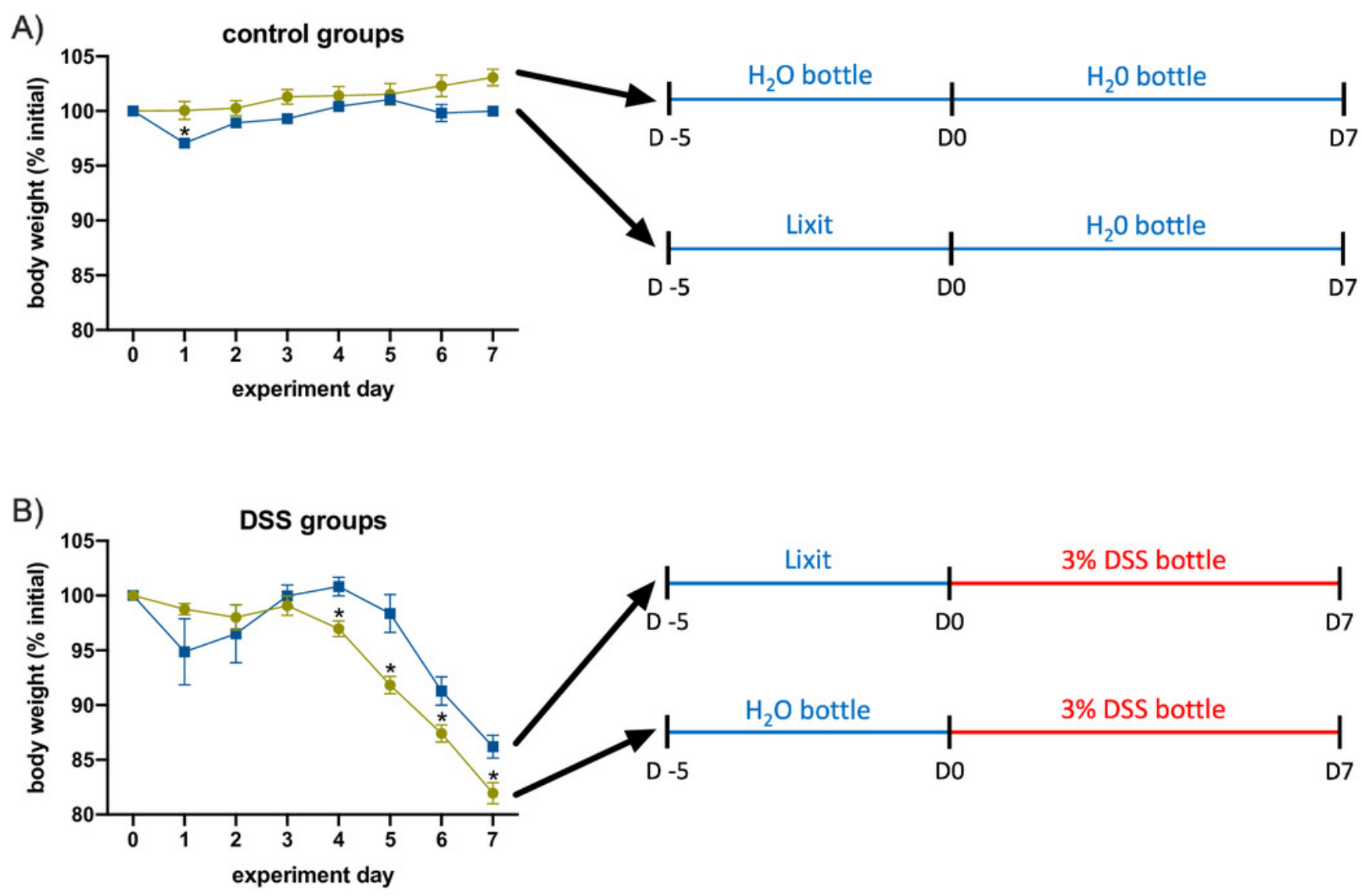